Trends in Cancer (forum article) Manuscript title:

\title{
Amphiregulin in cancer: new insights for translational medicine
}

$$
\text { Qixia } \mathrm{Xu}^{1,2}, \text { Paul } \text { Chiao }^{3} \text { and Yu Sun }{ }^{1,2,4}
$$

1. Key Lab of Stem Cell Biology, Institute of Health Sciences, Shanghai Institutes for

Biological Sciences, Chinese Academy of Sciences; Shanghai Jiaotong University,

School of Medicine, Shanghai, China 200031

2. Collaborative Innovation Center of Systems Biomedicine, Shanghai Jiaotong University

School of Medicine, Shanghai, China 200240

3. Department of Molecular and Cellular Oncology, The University of Texas MD Anderson

Cancer Center, Houston, TX, USA 77030

4. Department of Medicine and VAPSHCS, University of Washington, Seattle, WA, USA

98195

Keywords: amphiregulin, tumor microenvironment, immunosuppression, secretory phenotype, therapeutic resistance, translational medicine.

Correspondence to: Yu Sun, PhD, Shanghai Institutes for Biological Sciences (SIBS), Chinese Academy of Sciences (CAS) and Shanghai Jiaotong University School of Medicine (SJTUSM), 320 Yueyang Rd, Shanghai, China 200031. Tel: +86 21 54923302; Fax: +86 21 54923302; (e-mail: sunyu@sibs.ac.cn).

Disclosure: The authors disclose no potential conflicts of interest. 


\section{Abstract}

Although there is a large body of literature regarding amphiregulin (AREG) in human cancer, most knowledge focuses on its cell-autonomous functions in epithelial malignancies. Recent studies revealed that AREG is also present in the tumor microenvironment (TME) and contributes to therapeutic resistance. We discuss emerging concepts of AREG tumor biology and highlight their implications for translational medicine.

\section{AREG and mmunosuppressive Induction in the TME}

As an activating ligand of the epidermal growth factor receptor (EGFR), AREG is expressed in epithelial and mesenchymal cells and is physiologically essential for organ development and tissue homeostasis. The cancer cellautonomous functional roles of AREG such as stimulating growth, promoting invasion, enhancing metastasis and facilitating angiogenesis are well documented. However, new data showed that AREG is also expressed in the TME cells. Leukocyte populations including basophils, eosinophils, dendritic cells (DCs), mast cells, group 2 innate lymphoid cells (ILC2s), some tissueresident CD4+ regulatory $\mathrm{T}$ cells (Tregs) and a subset of tumor-infiltrating CD8+ $T$ cells comprehensively express AREG, suggesting a critical role of this factor in immune and/or inflammation-associated activities [1]. Although AREG is dispensable for Treg suppressor function, its elevated level in the

TME promotes Treg-associated immunosuppression, dampens local 
inflammation and mediates type 2 immune resistance, particularly upon activation of ILC2s and Tregs by interleukin-33 (IL-33) [2,3]. Further, AREG expression in the immune cells can be elicited by diverse stimuli including prostaglandin E2 (PGE2), insulin-like growth factor-1 (IGF-1), and transforming growth factor- $\beta$ (TGF- $\beta$ ), implying that AREG is produced upon activation of multiple cell types.

High local concentrations of AREG are present in the TME of epitheliumderived solid malignancies, where there is an enrichment of tumor infiltrating EGFR+ CD4+ Tregs. Given the presence of AREG-associated tissue-resident CD4+ Tregs in the stroma of these tumors, EGFR antagonists such as OGlcNAcylated macrophage migration inhibitory factor (MIF) used in brain tumors [4], may not only have a direct anti-cancer cell effect but also inhibit the function of local CD4+ Tregs, thereby mitigating immunosuppression and contributing to anti-cancer immune responses. However, the cellular sources of AREG and the mechanisms that regulate its expression remain largely unclear and future insights into these aspects could uncover new therapeutic targets for clinical intervention in multiple tumor types.

\section{Enhanced Production of AREG in the TME under Therapeutic Conditions}

DNA damage signals elicited by chemotherapy and/or radiation can be transmitted via master regulators including NF-KB and C/EBP $\beta$ to generate a powerful, highly conserved, cell-non-autonomous and senescence associated 
secretory phenotype (SASP) in the TME. Such a process is mediated by the DNA damage secretory program (DDSP) [5] (Figure 1A) and its downstream effectors comprise a large spectrum of extracellular proteins, including not only AREG but also the recently reported WNT16B, epiregulin (EREG), secreted frizzled-related protein 2 (SFRP2), hepatocyte growth factor (HGF), interleukin 8 (IL-8) and matrix metalloproteinases (MMPs). Together these soluble factors create a pro-angiogenic and pro-inflammatory microenvironmental niche that promotes a malignant phenotype, substantially fueling resistance and recurrence (Figure 1B).

Multiple signaling pathways are involved in the SASP, including but not limited to those engaging p38MAPK, macroH2A1 ( $\mathrm{H} 2 \mathrm{~A}$ histone family, member $\mathrm{Y}$ ), mTOR (mammalian target of rapamycin), and GATA4 (GATA binding protein 4) [6-9]. After genotoxic insults, each of these stroma-secreted factors exerts distinct pathological effects, while some can also interact with others in the TME, as illustrated by a recent study revealing functional interplay between WNT16B and SFRP2 [10]. It is thus important to determine if extracellular proteins physically associate with AREG, and how they mutually interact in the TME upon therapy.

Cancer patients who initially benefit from EGFR-targeted therapies frequently develop resistance. Understanding the mechanisms of resistance to EGFRtargeted therapies provides an opportunity to develop novel agents and 
treatment strategies. In PTEN-null triple negative breast cancer, AREGmediated activation of EGFR causes an increase in the levels of stromal fibronectin, which mediates invasion via interaction with integrin $\beta 1$, and engagement of PLK1 and BIRC5, genes that are essential for cancer cell survival but function independently of EGFR signaling [11]. In such case, AREG and other critical survival genes are distinctly uncoupled from regulation through the typical EGFR signaling axis, and can potentially mediate resistance to EGFR inhibitors in an EGFR-independent manner (Box 1).

\section{AREG: a Promising Clinical Biomarker and Therapeutic Target}

AREG holds a dual potential as a prognostic biomarker of treatment efficacy and as a therapeutic target for multiple tumor types. Serum levels of AREG are higher in hepatocellular carcinoma patients than in the healthy population, and the specificity of AREG in diagnostic screenings is better than that of $\alpha$ fetoprotein (AFP, a current biomarker for liver cancer), suggesting that AREG can be valuable as a novel serological marker [12]. Furthermore, mounting evidence indicates that AREG is a prominent target in epithelial cancers, specifically in AREG-overexpressing conditions. Several approaches have been employed to target AREG in different contexts, including in lung, breast, ovarian, colorectal and pancreatic malignancies. One approach involves RNA interference (RNAi)-mediated silencing of AREG to inhibit deoxycholic acid 
(DCA)-induced EGFR/MAPK (mitogen activated protein kinase)/STAT3 (signal transducer and activator of transcription 3) signaling and prevent DCAassociated colorectal cancer or pancreatic ductal adenocarcinoma [13]. AREG-neutralizing antibodies also show remarkable efficacy in controlling ovarian tumor growth, minimizing drug resistance and reducing metastatic potential [14]. A third approach involves the use of small molecule inhibitors of ADAM17 (a disintegrin and metalloproteinase 17) to suppress the shedding of AREG. The rationale is supported by a recent study showing that specific antiADAM17 antibodies are effective in ovarian cancer treatment [15]. Targeting the ADAM17-AREG-EGFR axis may arrest the development of resistance to anti-EGRF therapies.

\section{Concluding Remarks and Future Perspectives}

Despite exciting preclinical and early-stage clinical data, the majority of advanced clinical trials cannot reach their primary endpoints. Due to therapeutic resistance, a daunting and stubborn barrier to clinical success, cancer-associated mortality remains the highest among human pathologies. Tackling cancer resistance, especially the mechanisms acquired extrinsically and driven by treatment-activated or agent-remodeled TME, can help develop feasible complementary therapeutic strategies.

The functional roles of tumor stroma in disease initiation and progression is well appreciated, particularly the implication of tumor-infiltrating leukocytes 
(TILs) and cancer-associated fibroblasts (CAFs). The tumor-surrounding stroma contributes significantly to the behavior of treatment-refractory tumors. AREG is involved in cancer progression and has become the focus of several basic, translational and clinical investigations. Despite the role of AREG in human cancer, the cellular distribution and the mechanisms that regulate AREG expression are still debated.

Cancer cell-directed treatments should be ideally combined with therapies targeting the TME. Thorough understanding of pathological implications of a dysfunctional TME, specifically AREG and other TME-derived soluble factors, is critical. It is evident that timely and precise manipulation of the TME can affect cancer progression at different stages including the early formation of primary foci or the late metastatic colonization in distant organs.

Antibody therapeutics are a mainstay in cancer therapy since they directly target specific molecules and can serve as optimal vehicles for efficient delivery of cytotoxic agents, such as antibody-drug conjugates (ADCs) which are rapidly arising. Monoclonal antibodies can trigger antibody-dependent phagocytosis (ADP) or antibody-dependent cellular cytotoxicity (ADCC) and help eliminate antibody-bound target cells. Therefore, immunosuppression mediated by AREG-producing immune cells may be substantially mitigated by antibody therapy that reduces the number of AREG-positive leukocytes. In addition, clearance of therapeutically damaged stromal cells exhibiting 
senescent phenotypes may prevent the development of TME-induced resistance upon chemotherapy and/or radiation, as illustrated by recent work reporting the efficacy of "senolytic" pharmacological agents in vivo [16].

We envision that incorporation of TME-based therapeutic strategies into contemporary anti-cancer therapies can provide novel avenues to significantly improve therapeutic indexes and enhance cancer remission, as encouraged by the success of many emerging trials in clinical oncology.

\section{Acknowledgements}

This work was supported by a US DoD PCRP Idea Development Award (PC111703), National Natural Science Foundation of China (81472709) and National 1000 Youth Elites Research Program of China to Y. S.

\section{References}

1. Zaiss, D. M., Gause, W. C., Osborne, L. C., \& Artis, D. (2015). Emerging functions of amphiregulin in orchestrating immunity, inflammation, and tissue repair. Immunity, 42(2), 216-226, doi:10.1016/j.immuni.2015.01.020.

2. Arpaia, N., Green, J. A., Moltedo, B., Arvey, A., Hemmers, S., Yuan, S., et al. (2015). A Distinct Function of Regulatory T Cells in Tissue Protection. Cell, 162(5), 1078-1089, doi:10.1016/j.cell.2015.08.021.

3. Zaiss, D. M., van Loosdregt, J., Gorlani, A., Bekker, C. P., Grone, A., Sibilia, M., et al. (2013). Amphiregulin enhances regulatory $T$ cell-suppressive function via the epidermal growth factor receptor. Immunity, 38(2), 275-284, doi:10.1016/j.immuni.2012.09.023.

4. Zheng, Y., Li, X., Qian, X., Wang, Y., Lee, J. H., Xia, Y., et al. (2015). Secreted and O-GlcNAcylated MIF binds to the human EGF receptor and inhibits its activation. Nature Cell Biology, 17(10), 13481355, doi:10.1038/ncb3222.

5. Sun, Y., Campisi, J., Higano, C., Beer, T. M., Porter, P., Coleman, I., et al. (2012). Treatment-induced damage to the tumor microenvironment promotes prostate cancer therapy resistance through WNT16B. Nature Medicine, 18(9), 1359-1368, doi:Doi 10.1038/Nm.2890. 
6. Alspach, E., Flanagan, K. C., Luo, X. M., Ruhland, M. K., Huang, H., Pazolli, E., et al. (2014). p38MAPK Plays a Crucial Role in Stromal-Mediated Tumorigenesis. Cancer Discov, 4(6), 716-729, doi:10.1158/2159-8290.CD-13-0743.

7. Chen, H., Ruiz, P. D., McKimpson, W. M., Novikov, L., Kitsis, R. N., \& Gamble, M. J. (2015). MacroH2A1 and ATM Play Opposing Roles in Paracrine Senescence and the SenescenceAssociated Secretory Phenotype. Mol Cell, 59(5), 719-731, doi:10.1016/j.molcel.2015.07.011.

8. Laberge, R. M., Sun, Y., Orjalo, A. V., Patil, C. K., Freund, A., Zhou, L., et al. (2015). MTOR regulates the pro-tumorigenic senescence-associated secretory phenotype by promoting IL1A translation. Nature Cell Biology, 17(8), 1049-1061, doi:10.1038/ncb3195.

9. Kang, C., Xu, Q., Martin, T. D., Li, M. Z., Demaria, M., Aron, L., et al. (2015). The DNA damage response induces inflammation and senescence by inhibiting autophagy of GATA4. Science, 349(6255), aaa5612, doi:10.1126/science.aaa5612.

10. Sun, Y., Zhu, D., Chen, F., Qian, M., Wei, H., Chen, W., et al. (2016). SFRP2 augments WNT16B signaling to promote therapeutic resistance in the damaged tumor microenvironment. Oncogene, doi: 10.1038/onc.2015.494.

11. Kappler, C. S., Guest, S. T., Irish, J. C., Garrett-Mayer, E., Kratche, Z., Wilson, R. C., et al. (2015). Oncogenic signaling in amphiregulin and EGFR-expressing PTEN-null human breast cancer. Mol Oncol, 9(2), 527-543, doi:10.1016/j.molonc.2014.10.006.

12. Han, S. X., Bai, E., Jin, G. H., He, C. C., Guo, X. J., Wang, L. J., et al. (2014). Expression and clinical significance of YAP, TAZ, and AREG in hepatocellular carcinoma. Journal of Immunology Research, 2014, 261365, doi:10.1155/2014/261365.

13. Nagathihalli, N. S., Beesetty, Y., Lee, W., Washington, M. K., Chen, X., Lockhart, A. C., et al. (2014). Novel mechanistic insights into ectodomain shedding of EGFR Ligands Amphiregulin and TGFalpha: impact on gastrointestinal cancers driven by secondary bile acids. Cancer Res, 74(7), 2062-2072, doi:10.1158/0008-5472.CAN-13-2329.

14. Carvalho, S., Lindzen, M., Lauriola, M., Shirazi, N., Sinha, S., Abdul-Hai, A., et al. (2015). An antibody to amphiregulin, an abundant growth factor in patients' fluids, inhibits ovarian tumors. Oncogene, doi:10.1038/onc.2015.93.

15. Richards, F. M., Tape, C. J., Jodrell, D. I., \& Murphy, G. (2012). Anti-tumour effects of a specific antiADAM17 antibody in an ovarian cancer model in vivo. Plos One, 7(7), e40597, doi:10.1371/journal.pone.0040597.

16. Chang, J., Wang, Y., Shao, L., Laberge, R. M., Demaria, M., Campisi, J., et al. (2016). Clearance of senescent cells by ABT263 rejuvenates aged hematopoietic stem cells in mice. Nature Medicine, 22(1), 78-83, doi:10.1038/nm.4010.

\section{Figure Legends}

Figure 1. AREG is significantly produced upon genotoxic damage to human stromal cells and promotes therapeutic resistance to surviving 


\section{cancer cells.}

(A) Genome-wide expression analysis of primary normal human prostate stromal cells exposed to DNA damaging agents: $\mathrm{H}_{2} \mathrm{O}_{2}$, hydrogen peroxide (0.6 $\mathrm{mM}$ for 2 hours); Bleo, bleomycin (100 ug/ml for 6 hours); and Rad, ionizing radiation (10 Gy overall exposure) to allow development of the DNA damage secretory program (DDSP)-driven full spectrum senescence associated secretory phenotype (SASP) as a chronic response. A list of top upregulated human genes encoding extracellular or secreted factors is shown, average expression fold change $\geq 3.5$ by comparison of post- vs pre-treatment samples. AREG is among the top overexpressed genes [5]. (B) Working model for TME-mediated therapeutic resistance acquired upon genotoxic treatments. Chemo/radiation causes apoptosis in subsets of cancer cells by eliciting a DNA damage response (DDR), while cancer cells with DDR deficiency (DDR-incompetent or DDR-insensitive) may escape from such insults. Simultaneously, senescence is induced in stroma adjacent to epithelium-surrounding glands (such as those in prostate or breast tissues), upon DDR-mediated signaling. The DDSP is usually characterized by a spectrum of autocrine- and paracrine-acting proteins. The soluble factors reinforce the senescent phenotype in damaged stromal cells, enhance cancer cell repopulation, increase occurrence of tumor relapse and distant metastasis. A handful of TME-released factors including WNT16B and AREG hold the potential to serve as both a serum biomarker to determine treatment index, 
and a therapeutic target to minimize the TME-conferred therapeutic resistance. DDR, DNA damage response; ECM, extracellular matrix; TME, tumor microenvironment. Color images of (A) adapted from reference [5] with permission from Nature Medicine, copyright 2012.

\section{Box 1}

The advent of targeted therapy has revolutionized cancer treatment by interfering with single or multiple signaling pathways of malignant cells. Although monoclonal antibodies such as Cetuximab target the ligand-binding domain of EGFR, resistance to EGFR-directed therapy can be mediated though activation of extracellular signal-regulated kinase 1/2 (ERK1/2) which functionally bypasses EGFR. In contrast to such well understood mechanisms, the role of extracellular factors is less known but important because upregulation of EGFR ligands, such as AREG, may also cause receptor activation and dictate drug sensitivity. In the setting of genotoxic treatments including chemotherapy and radiation, enhanced AREG production by the tumor-adjacent stroma holds can contribute to enhance cancer resistance. Such resistance is driven by TME-derived soluble factors that mediate signaling via the plasma membrane receptor of cancer cells, and thus belong to the class of acquired and extrinsic resistance, in contrast to the commonly discussed de novo and intrinsic resistance. 


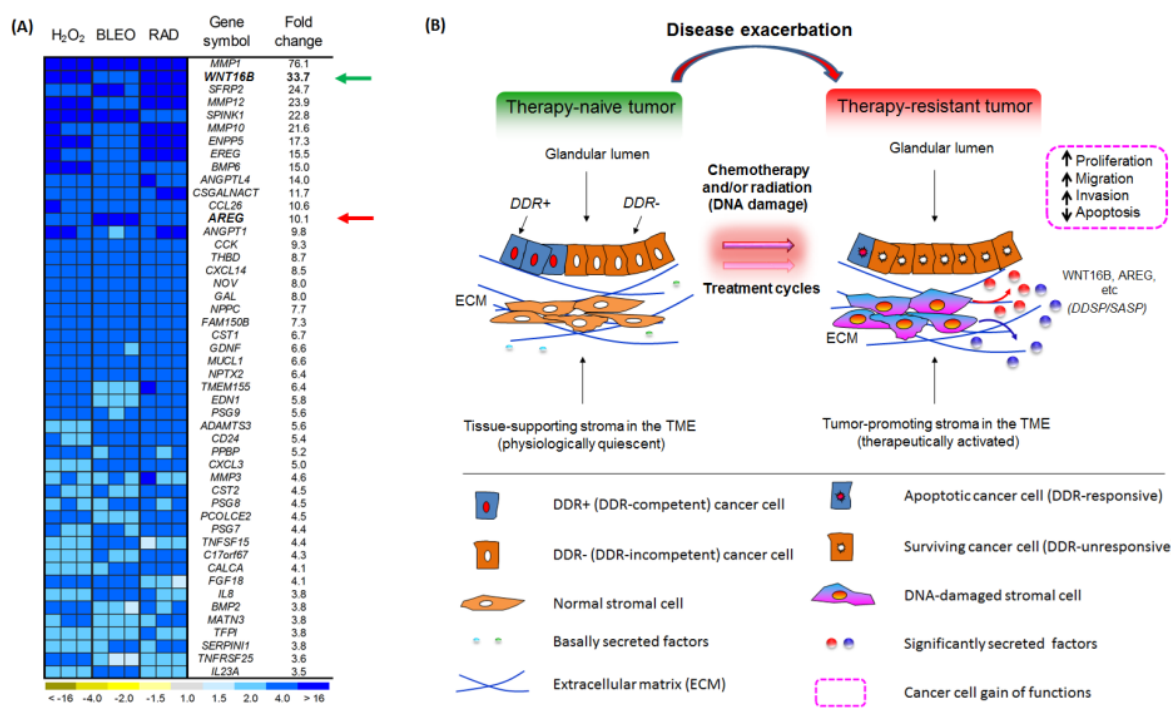

